\title{
Museus, Turismo e Sociedade: análise bibliométrica da produção científica ibero-americana
}

\author{
Luciana Ferreira da Costa
}

\section{Resumo:}

Este artigo objetiva analisar a produção científica sobre "Museus, Turismo e Sociedade" dos artigos científicos de três Dossiês dedicados a esta temática e publicados pela Revista Iberoamericana de Turismo nos anos de 2014, 2015 e 2017. Caracteriza-se por sua natureza como bibliográfico, sob abordagem metodológica qualitativa com aporte quantitativo. A coleta de dados se deu a partir do levantamento dos artigos publicados. Foram identificados 9,10 e 13 artigos, respectivamente, nos Dossiês publicados nos anos de 2014, 2015 e 2017. Ao todo 32 artigos publicados. $A$ análise de dados se deu através da análise bibliométrica e de conteúdo por categorias temáticas sobre os artigos. Conclui com a importância das relações entre Museus e Turismo na sociedade contemporânea e a contribuição dos Dossiês da revista para a compreensão e o avanço dessas relações.

\section{Palavras-chave:}

Museus; Turismo; Bibliometria; Revista Iberoamericana de Turismo.

\begin{abstract}
:
This paper aims to analyze the scientific production on "Museums, Tourism and Society" of the scientific articles of three Dossiers dedicated to this theme and published by the Ibero-American Tourism Journal in the years of 2014, 2015 and 2017. It is characterized by its nature as bibliographical, under qualitative methodological approach with quantitative contribution. Data collection was based on the survey of articles. We identified 9, 10 and 13 articles, respectively, in the Dossiers published in the years 2014, 2015 and 2017. In all 32 articles published. The analysis of data was done through bibliometric analysis and content by thematic categories on the articles. It concludes with the importance of the relations between Museums and Tourism in contemporary society and the contribution of the Dossiers of the journal to the understanding and the advance of these relations.
\end{abstract}

\section{Keywords:}

Museums; Tourism; Bibliometry; Ibero-American Tourism Journal.

\section{Introdução}

Desde a criação dos museus e o advento do Turismo, as relações entre os museus e o Turismo vêm contribuindo para o reconhecimento mútuo entre as culturas e para o respeito à diversidade cultural, promovendo experiências de aprendizagem, conhecimento e lazer. Nesse sentido, o então Secretário Geral do Conselho Internacional de Museus (ICOM), Manus Brinkman, declarou que o Turismo pode ser uma vantagem complexa:se existem milhares de museus para as pessoas e a elas se dirigem, são necessários turistas para visitá-los, não apenas por motivos econômicos, contudo, também para lhes transmitir conhecimentos sobre as sociedades e a história e, assim, surpreendê-los (BRINKMAN, 2000). $\mathrm{Na}$ Declaração do ICOM e da Federação Mundial de Amigos dos Museus

I Doutora em História e Filosofia da Ciência pela Universidade de Évora, Portugal. Professora do Departamento de Ciência da Informação da Universidade Federal da Paraíba, Brasil. 
(FMAM) para o Turismo Cultural Sustentável em todo o mundo, publicada em dezembro de 2007 em Paris, França, o ICOM e a FMAM consideraram tornar realidade a proposta da "Carta de Princípios para Museus e Turismo Cultural", formulada pelo ICOM durante uma conferência na Bolívia e no Peru no ano de 2000, onde alguns dos seus preceitos são aqui destacados:

O patrimônio cultural não pode se tornar um produto de consumo nem sua relação com o visitante pode ser superficial. Se o turista conseguir identificar-se com o patrimônio, poderá valorizar e preservar a importância e, portanto, tornar-se um aliado dos museus. [...] No que diz respeito ao turismo cultural, os museus devem incentivar a participação ativa das comunidades locais no planejamento da gestão do patrimônio e das operações dos locais turísticos. [...] Os museus devem incentivar as comunidades a administrar seu patrimônio cultural, para o qual devem incentivar um treinamento adequado. [...] É importante planejar passeios usando programas temporários que são restritos para satisfazer os períodos de lazer dos habitantes locais e oferecer alternativas para turistas estrangeiros. Os museus e o turismo cultural devem incentivar a interação entre os visitantes em um quadro de respeito aos valores e à hospitalidade oferecida (ICOM, 2007, on-line, tradução nossa).

Como a terceira maior atividade econômica mundial, o Turismo tem um alcance global. Os museus, que existem em todo o mundo, afetam os seus visitantes principalmente nas regiões de destinos turísticos. Isso dá aos museus uma forte chance de ter sucesso em seus esforços educacionais para substituir ideias ultrapassadas sobre cultura, gestão e desenvolvimento sustentável. Os museus devem ser cada vez mais integrados aos conceitos de Turismo, para garantir que eles tenham influência sobre agentes econômicos e governos nos processos de planejamento comunitário, público e empresarial da educação e cultura. O ICOM e a FMAM, juntamente com a Organização Mundial do Turismo (OMT), vêm conscientizando os museus e amigos de museus ao redor do mundo sobre o tema do Turismo Cultural Sustentável, incluindo o Dia Internacional dos Museus (ICOM, 2007).

Nesse sentido, este artigo se enquadra na articulação entre Museus, Turismo e Sociedade, como encontrado nas pistas do ICOM, da FMAM, da OMT e de autores brasileiros e portugueses das áreas da Museologia e Patrimônio e do Turismo, a exemplo de Teresa Scheiner (2017) e Alexandra Gonçalves (2017). Estas duas autoras, em seus recentes artigos científicos, comentam as ressonâncias das estratégias mundiais no campo do patrimônio e dos museus sobre a atividade turística, partindo da noção que existem novos paradigmas por muito a serem explorados aos quais os museus não podem permanecer indiferentes, principalmente os paradigmas relacionados ao turismo cultural e ao turismo de massa.

Diante do exposto, este artigo tem como objetivo analisar a produção científica sobre "Museus, Turismo e Sociedade" dos artigos científicos de três Dossiês dedicados a esta temática e publicados pela Revista lberoamericana de Turismo (RITUR) nos anos de 20I4, de 2015 e de 2017 . Refere-se às conclusões de um estudo que se caracteriza por sua natureza como bibliográfico, sob abordagem metodológica qualitativa com aporte quantitativo, e análise bibliométrica e de conteúdo por categorias temáticas sobre os artigos científicos. 
Este artigo se desenvolve na esteira da importância das pesquisas bibliométricas sobre a produção científica das diversas áreas do conhecimento, mais notadamente, por tradição, a Ciência da Informação e a Biblioteconomia. Vale ressaltar as pesquisas bibliométricas sobre artigos científicos publicados nos mais expressivos e atualizados canais da comunicação científica na atualidade: os periódicos científicos eletrônicos de acesso aberto ${ }^{2}$.

Os periódicos científicos eletrônicos se popularizaram na década de 1990 com o advento da Internet. Inúmeras são as vantagens dos periódicos científicos eletrônicos: menor custo; facilidade de avaliação de trabalhos para publicação no formato on-line, na versão blind review by peers (revisão às cegas por pares) e ad hoc (sob designação específica de especialistas para cada avaliação); maior divulgação; proteção do acervo; agilidade na publicação; facilidade de acesso; apresentação do documento adotando novos padrões; diversidade de formatos/mídias (sons, imagens, vídeos, hipertexto, etc.); e recuperação da informação automatizada e até personalizada, referindo-se da indexação à busca e localização de informações (CRESPO, 2005; MAIA, 2005).

No contexto da publicação de periódicos científicos na Internet, dois modelos de acesso se destacam: os periódicos por assinatura ou pagos, denominados periódicos de acesso restrito; e os periódicos com acesso aberto ou gratuito à informação científica.

Os periódicos de acesso aberto surgiram com o Movimento de Acesso Aberto. O Movimento de Acesso Aberto, iniciado na década de 1990, configura-se como um amadurecimento da comunicação científica em que comungam atores individuais e institucionais em prol da disseminação e da visibilidade de sua produção científica em acesso aberto para evolução das áreas científicas. $O$ Movimento de Acesso Aberto se constitui como o acontecimento "mais interessante e talvez importante de nossa época no que se refere à comunicação científica", pois ele é representativo da "mudança provocada no sistema tradicional e profundamente arraigado de comunicação do conhecimento científico", sendo o Brasil pioneiro na promoção deste movimento, seja por periódicos científicos seja por repositórios institucionais que disponibilizam seus conteúdos de forma gratuita (MUELLER, 2006, p. 27).

Pretende-se, portanto, desvelar aqui a produção científica sobre as relações prático-epistemológicas entre museus e Turismo na sociedade contemporânea no âmbito da América Latina e Europa lbérica, a partir do que foi publicado nos Dossiês temáticos da RITUR, como um periódico científico de acesso aberto de reconhecimento internacional.

Até porque, os periódicos científicos se revelam como fontes necessárias para todas as áreas do conhecimento científico, e especificamente para a área da Museologia, destacando para escoamento da sua produção científica certa frequência de periódicos do campo do Turismo (COSTA; NUNES; LOPES, 20I5).

2 No Brasil, diante da importância dos periódicos científicos, o denominado sistema Qualis Periódicos vem sendo utilizado para classificar a produção científica dos programas de pós-graduação stricto sensu no que se refere aos artigos publicados em periódicos científicos. Tal processo foi concebido para atender um dos requisitos do sistema de avaliação nacional dos programas de pós-graduação pela Coordenação de Aperfeiçoamento de Pessoal de Nível Superior (CAPES). Como resultado, disponibiliza uma lista com a classificação dos periódicos utilizados pelos programas de pós-graduação para o escoamento da sua produção (CAPES, 2018). 
Museus, Turismo, Sociedade:

análise bibliométrica da produção científica íbero-americana

\section{A Revista Ibero-americana de Turismo (RITUR)}

A RITUR é um periódico científico eletrônico de acesso aberto de iniciativa do Observatório Transdisciplinar de Pesquisas em Turismo (OTPT) da Universidade Federal de Alagoas (UFAL), Brasil, e do Laboratori Multidisciplinar de Recerca en Turisme (LMRTur) da Facultat de Turisme da Universitat de Girona (UdG), Espanha, que nasce no ano de 2010 do convênio de cooperação internacional de pesquisa e desenvolvimento em Turismo entre estas instituições, com o financiamento da Agencia Española de Cooperación Internacional para el Desarrollo (AECID), Espanha, e com a composição de uma experiente equipe editorial formada por pesquisadores nacionais e internacionais ${ }^{3}$. Por sua origem, a grafia do título da revista, dessa forma, atende à língua espanhola por convenção (RITUR, 20I8e).

O OTPT da UFAL, criado em 20I I, tem como objetivo articular diferentes áreas em torno da temática do Turismo, contemplando inter-relações com a cultura, a hospitalidade, as transformações sociais, o desenvolvimento econômico, a política e o processo de comunicação. Promovendo investigações em cooperações nacionais e internacionais, o OTPT, considerando o caráter múltiplo de relações disciplinares a partir do Turismo, possibilita a compreensão dos mecanismos de construção e consolidação do fenômeno do Turismo, abrangendo sua produção e seu consumo na sociedade (OTPT, 20।8).

A formação de um grupo de pesquisas em Turismo na UdG remonta o ano de 1997. Desde sua criação, o LMRTur da Facultat de Turisme da UdG tem se dedicado às práticas de investigação em Turismo nesta universidade, também promovendo cooperações nacionais e internacionais e subsidiando a pós-graduação stricto sensu da UdG, no que se refere ao seu Doutorado em Turismo, em funcionamento desde o ano de 2005, e os seus três mestrados: o Màster en Direcció i Planificació del Turisme; o Màster en Turisme Cultural; e o Erasmus Mundus European Master's in Tourism Management (UDG, 2018).

Como financiadora do projeto de cooperação internacional entre a UFAL e a UdG, entre os anos de 2009 e 2010, que culminou na criação da RITUR, a AECID é o principal órgão de gestão da Cooperação Espanhola com mais de 30 países, com o objetivo de combater a pobreza e promover o desenvolvimento humano sustentável, a coesão social e a igualdade de direitos para as pessoas nos países parceiros. A AECID funciona sob a tutela do Ministério das Relações Exteriores e Cooperação do Governo da Espanha. Desde o ano de 20II, a AECID não financia mais projetos que incluam o Brasil, por considerá-lo um dos 10 países mais ricos do mundo, retirando o país de sua agenda de projetos custeados (AECID, 20I8).

Posteriormente, a RITUR passou a contar com a colaboração da Rede de Pesquisa e (In)Formação em Museologia e Patrimônio (REDMUS) do Programa de Pós-Graduação em Ciência da informação (PPGCl) da Universidade Federal da Paraíba (UFPB), Brasil, e do Instituto de História Contemporânea - Grupo de Investigação Ciência, Estudos de História, Filosofia e Cultura Científica (IHC-CEHFCi) da Universidade de Évora (UÉvora), Portugal, a partir da

3 Vale destacar o trabalho do Conselho Editorial da RITUR, formado por 18 profissionais/docentes/ pesquisadores doutores, das seguintes instituições nacionais e estrangeiras: Universidade Anhembi Morumbi, Brasil; Universidad de La Laguna, Espanha;UFAL, Brasil;UdG, Espanha; Instituto Politécnico de Tomar, Portugal; Universidade de São Paulo, Brasil; Universidade Federal do Ceará, Brasil; Diretoria Regional de Cultura do Algarve, Portugal; Escola Superior de Hotelaria e Turismo do Estoril, Portugal; Universidade Federal do Rio Grande do Norte, Brasil; Universidade Paulista, Brasil; e Universidade Federal do Paraná, Brasil (RITUR, 2018c). 
publicação do seu primeiro número especial no ano de 2014 (RITUR, 20I8e). A REDMUS do PPGCI da UFPB foi criada em 20I4, com o objetivo de promover a investigação/formação/divulgação científica sobre a área da Museologia e Patrimônio em perspectiva transcontinental, tendo como eixo transversal as múltiplas práticas (in)formacionais sobre espaços e saberes museológicos. Pela vinculação ao PPGCl da UFPB, ficam evidentes as relações disciplinares promovidas pela REDMUS envolvendo a área da Ciência da Informação, bem como outras áreas como História da Ciência e Turismo.A REDMUS mantém cooperação nacional com a UFAL e cooperação internacional com o Instituto Politécnico de Leiria (IPLeiria), Portugal, e a UÉvora (REDMUS, 20I8).

O IHC-CEHFCi da UÉvora iniciou-se em 1994, denominado de Centro de Estudos de História e Filosofia da Ciência (CEHFCi), por meio de um grupo de estudiosos de diversas áreas científicas. Sua evolução ao longo dos anos e a percepção da necessidade de afirmação da recente área da História e Filosofia da Ciência em Portugal foram motivadoras para que em 1998 o grupo se candidatasse a centro de estudos no âmbito da Fundação para Ciência e Tecnologia (FCT), Portugal, onde se enquadra desde então, sendo uma unidade científica do Programa de Doutoramento em História e Filosofia da Ciência com Especialidade em Museologia da UÉvora, Portugal. No ano de 2015 passou a ser reconhecido sob a denominação IHC-CEHFCi, mantendo sua agenda, estratégias e objetivos (IHC-CEHFCl, 20I8).

De periodicidade semestral ininterrupta, a RITUR tem por objetivo divulgar trabalhos científicos que representam contribuição para o desenvolvimento de novos conhecimentos entre pesquisadores, docentes, discentes e profissionais em Turismo, Hospitalidade, Museologia e Patrimônio, História, Geografia, Lazer e áreas afins, independente de sua vinculação profissional e local de origem, priorizando diálogos abertos e abordagens interdisciplinares a transdisciplinares na perspectiva do compartilhamento e difusão de conhecimentos a partir do que é produzido na América Latina e Europa Ibérica. Publica artigos científicos, pontos de vista, entrevistas, ensaios e resenhas, além de seus editoriais e expedientes, nas línguas portuguesa, inglesa, espanhola e catalã (RITUR, 20I8e).

Enquanto um periódico eletrônico de acesso aberto, a RITUR está hospedada no Serviço de Editoração Eletrônica de Revistas da UFAL ${ }^{4}$, mantido pela Biblioteca Central desta universidade, utilizando o Sistema Eletrônico de Editoração de Revistas (SEER) desenvolvido pelo Instituto Brasileiro de Informação em Ciência e Tecnologia (lbict) (IBICT, 20I8, on-line).

Com indexação internacional ${ }^{5}$, a RITUR disponibiliza seu conteúdo de modo gratuito, assumindo o compromisso com a OAl, sem cobrança para publicação de trabalhos originais. O OAl é um protocolo internacional que define um mecanismo para coleta de registros de metadados em repositórios, como iniciativa para aumentar e promover normas de interoperabilidade que visam facilitar a disseminação e o acesso eficiente de conteúdos na Internet (OAI,

4 Disponível em: http://www.seer.ufal.br/index.php/ritur.

5 A RITUR se encontra indexada nos seguintes sistemas, bases de dados e repositórios: Sistema Regional de Información en Línea para Revistas Científicas de América Latina, el Caribe, España y Portugal (LATINDEX); Directory of Open Access Journals (DOAJ); Sumários de Revistas Brasileiras, sob responsabilidade da Fundação de Pesquisas Científicas de Ribeirão Preto (Sumarios.org); Serviço de Editoração Eletrônica de Revistas da UFAL; Catàleg de la Biblioteca Digital de la UdG; Catàleg Col lectiu de les Universitats de Catalunya (CCUC); Electronic Journals \& Newsletter, Georgetown University Library (Newjour); Google Acadêmico; EBSCO Information Services; Red Iberoamericana de Innovación y Conocimiento Científico (REDIB); Rede Cariniana do Ibict; e Portal Oasisbr do Ibict (RITUR, 20I8d). 
2018), em acordo com o Movimento de Acesso Aberto.

A RITUR ainda está classificada no sistema Qualis Periódicos (Quadriênio 20I3-20I6) da Plataforma Sucupira da Coordenação de Aperfeiçoamento de Pessoal de Nível Superior (CAPES) do Ministério da Educação (MEC) do Governo do Brasil nos seguintes estratos: BI - Planejamento Urbano e Regional/Demografia; B3 - Administração Pública e de Empresas, Ciências Contábeis e Turismo; B3 - Ciências Ambientais; B3 - Educação; B3 - Interdisciplinar; B4 - Geografia; B4 - Antropologia/Arqueologia; B5 - Comunicação e Informação; B5 - Sociologia; e C - Biodiversidade (CAPES, 20I8).

Como um periódico científico consolidado, a RITUR, mesmo criada no ano de $2010 \mathrm{com}$ seu sistema on-line aberto para submissões, publicou seu primeiro número em janeiro de $20 \mathrm{II}$ (o volume I, número I de $20 \mathrm{I}$ ). Desde o seu lançamento, a RITUR publicou 20 edições, sendo destas I5 números correntes, respeitando a periodicidade semestral, e 5 números especiais. No momento, encontra-se publicada a sua última edição, o volume 8 , número 1 de 2018. Das 20 edições mencionadas, foram publicados ao todo: 184 artigos científicos, 5 pontos de vista, 2 entrevistas, I ensaio e 10 resenhas, além de 20 editoriais e 20 expedientes (RITUR, 20I8b).

A maior parte dos itens publicados na RITUR teve autores com a titulação de Doutor, pois, pelas normas do periódico, só é possível nele publicar trabalhos com no mínimo um autor com o título de Mestre ou Doutor (RITUR, 2018a).

Ademais, como dado de abrangência e repercussão da RITUR, constam registrados no sistema do periódico até 3I de junho de 20 I8: I755 usuários cadastrados como leitores e 309 usuários cadastrados como autores que colaboraram com submissões de trabalhos já publicados, brasileiros e estrangeiros (informação verbal) ${ }^{6}$.

\section{Os Dossiês Temáticos sobre "Museus, Turismo e Sociedade"}

Desde sua criação em 2010, a RITUR publicou cinco números especiais, sendo que três deles voltados à relação entre as disciplinas do Turismo e da Museologia por demanda da comunidade científica e decisão do seu Conselho Editorial: Dossiê Número I, Dossiê Número 2 e Dossiê Número 3, lançados respectivamente nos anos de 2014 , de 2015 e de 2017, todos sobre a temática "Museus, Turismo e Sociedade" (RITUR, 2018b).

Os outros dois números especiais se referiram às temáticas "Turismo e Políticas Urbanas" e "Destino Turístico Competitivo en los Albores del Siglo XXI”, lançados, por sua vez, respectivamente, nos anos de 2015 e de 2016 (RITUR, 20I8b).

Os três Dossiês sobre a temática "Museus, Turismo e Sociedade" foram forjados pela iniciativa conjunta, intercontinental e transnacional, da REDMUS do PPGCI da UFPB, Brasil, do IHC-CEHFCi da UÉvora, Portugal, bem como do Programa de Doutoramento em História e Filosofia da Ciência com Especialidade em Museologia desta universidade portuguesa, como organizadores das publicações, contando com a supervisão e o apoio editorial científico do OTPT da UFAL, Brasil, e do LMRTur da Facultat de Turisme da UdG, Espanha, por meio da articulação e colaboração entre as instituições e docentes pesquisadores envolvidos (COSTA; NUNES, 2017).

6 Informação fornecida pelo Editor Gerente da RITUR, Alan Curcino Pedreira da Silva, em João Pessoa, Brasil, em 31 de junho de 2018. 
Os três Dossiês sobre a temática "Museus, Turismo e Sociedade" da RITUR tiveram como Editoras responsáveis a Professora do Departamento de Ciência da Informação da UFPB, Brasil, com Doutorado em História e Filosofia da Ciência com Especialidade em Museologia pela UÉvora, Portugal, Líder da REDMUS do PPGCI da UFPB, Brasil; e a Professora Catedrática do Departamento de História da UÉvora, Portugal, com Doutorado em História Cultural Moderna e Contemporânea pela UÉvora, Portugal, Diretora do IHC-CEHFCi e Diretora do Programa de Doutoramento em História e Filosofia da Ciência com Especialidade em Museologia ambos também da UÉvora, Portugal (RITUR, 20I8b).

A proposta de publicação dos três Dossiês sobre a temática "Museus, Turismo e Sociedade" da RITUR foi de contribuir para as diversas áreas dedicadas a reflexões sobre os espaços museais e o conhecimento museológico, enfocando, sobretudo, uma perspectiva a partir do Turismo. $\mathrm{Na}$ realização dos Dossiês foram encontradas muitas análises sob dimensões sociais, antropológicas, históricas, políticas e econômicas, evocando, transversalmente, os conceitos de cultura, memória, patrimônio e educação na constelação das relações entre museus, turismo e sociedade apresentadas pelos autores (COSTA; NUNES, 2017).

Os três Dossiês sobre a temática "Museus, Turismo e Sociedade" da RITUR contaram com autores consagrados nacionais e internacionais, pesquisadores, professores e coordenadores de programas de pós-graduação, alguns com reconhecidos cargos públicos de direção e de representação institucional em organismos de classe - do Brasil, Espanha e Portugal, advindos principalmente das áreas da Museologia, Patrimônio, Turismo, Ciência da Informação e História, em acordo com o objetivo da RITUR de promover diálogos, compartilhamentos e difusão de conhecimentos interdisciplinares à transdisciplinares, a partir do que é produzido na América Latina e Europa Ibérica (COSTA; NUNES, 2017).

\section{Percurso Metodológico}

Considerando o objetivo deste artigo, de analisar a produção científica sobre "Museus, Turismo e Sociedade" nos artigos científicos dos Dossiês temáticos publicados pela RITUR, nos anos de 20I4, de 2015 e de 2017, trata-se de um estudo que se caracteriza por sua natureza como bibliográfico, sob abordagem metodológica quantitativa com aporte qualitativo (RICHARDSON, 1999). A pesquisa bibliográfica consiste no exame da literatura científica para levantamento e análise do que já foi produzido sobre determinado tema. Engloba - levantamento de toda a bibliografia já publicada, de modo a possibilitar ao pesquisador contato direto com o instrumental bibliográfico sobre tema ou assunto de interesse (MARCONI; LAKATOS, 2009) visando a sua exaustividade e pertinência (ALBARELLO et al., I997).

A coleta de dados se deu a partir do levantamento bibliográfico dos artigos científicos publicados nos três Dossiês temáticos sobre "Museus, Turismo e Sociedade" da RITUR em sua página oficial.

Para análise dos dados, foi utilizada a bibliometria aliada à análise de conteúdo por categorias temáticas, lançando mão de estatística básica e descritiva por utilização de frequências absolutas e percentuais.

Apesar da sua forte característica quantitativa, a evolução dos estudos bibliométricos lhe conferiu também a possibilidade "de atribuir sentido aos da- 
Museus, Turismo, Sociedade:

análise bibliométrica da produção científica íbero-americana

dos, qualificando-os para que possam ter melhor uso por parte de políticas de ciência e tecnologia (C\&T)", áreas de conhecimento, centros/grupos de pesquisas, dentre outros contextos (SANTOS; KOBASHI, 2009, p. I59-I60).

Este estudo ora relatado se utiliza da abordagem bibliométrica, contudo, acompanhando a vertente de trabalhos atuais realizados por autores da Ciência da Informação "que realizam uma leitura desses dados [quantitativos] à luz de elementos do contexto sócio-histórico em que a atividade científica é produzida" (ARAÚJO, 2006, p. 25).

Por sua vez, a análise de conteúdo utilizada é um conjunto de técnicas de análise das comunicações de modo a obter, por meio de procedimentos sistemáticos e objetivos de descrição do conteúdo das mensagens, indicadores que possibilitem inferir conhecimentos relativos às mensagens (BARDIN, I 979), portanto, configura-se como metodologia de análise fundamental para, entre outras questões, estudos de tendências (RICHARDSON, 1999).

A utilização da análise de conteúdo requer a criação de categorias relacionadas ao objeto de estudo.A categorização "é uma operação de classificação de elementos constitutivos de um conjunto, por diferenciação e, seguidamente, por reagrupamento" segundo a tipologia. As categorias são rubricas ou classes que englobam "um grupo de elementos (...) sob um título genérico, agrupamento esse efectuado em razão dos caracteres comuns destes elementos" (BARDIN, I 979, p. I I7). Neste estudo optou-se pela utilização da análise categorial temática.

A seguir, apresentam-se os resultados da análise bibliométrica deste estudo, sob as seguintes categorias temáticas determinadas: Quantidade de artigos científicos por Dossiê; Idiomas utilizados nos artigos; Quantidade de autores por Dossiê; Distribuição de autoria única e múltipla; Formação acadêmica dos autores; Instituições em que os autores realizaram a sua formação acadêmica; Instituições de vinculação dos autores; Temas dos artigos científicos por Dossiê; Frequência de Temas dos artigos científicos; Palavras-chave mais citadas;Tipologia documental referenciada; e Autores mais referenciados.

\section{Resultados e Análises}

Aqui, são apresentados os resultados e as análises do estudo sobre a produção científica sobre os artigos científicos dos Dossiês temáticos "Museus, Turismo e Sociedade" publicados pela RITUR, nos anos de 20I4, de 2015 e de 2017, através das categorias temáticas.

Como primeira categoria temática, Quantidade de artigos científicos por Dossiê, foram identificados 9 (28\%), I0 (3I\%) e 13 (4I\%) artigos científicos, respectivamente, nos Dossiês publicados nos anos de 20I4, de 2015 e de 2017. Ao todo 32 (100\%) artigos publicados.

Sobre a segunda categoria temática, Idiomas utilizados nos artigos, foi identificado o uso das línguas portuguesa e espanhola, algo esperado diante do objetivo da RITUR de publicar a produção científica a partir do que é produzido na América Latina e Europa Ibérica.

Dos 9 (I00\%) artigos científicos do Dossiê Número I de 20I4, 6 (67\%) foram publicados na língua portuguesa e 3 (33\%) foram publicados na língua espanhola. Dos 10 (100\%) artigos científicos do Dossiê Número 2 de 2015, 9 (90\%) foram publicados na língua portuguesa e I (10\%) foi publicado na língua espanhola. E, dos 13 (100\%) artigos científicos do Dossiê Número 3 de 2017, 10 (77\%) foram publicados na língua portuguesa e 3 (23\%) foram publicados na 
língua espanhola.

Assim, do total de 32 (100\%) de artigos científicos publicados nos três Dossiês, 25 (78\%) foram publicados majoritariamente na língua portuguesa e 7 (22\%) foram publicados na língua espanhola, ressaltando-se que os artigos científicos publicados na língua portuguesa tiveram origem tanto do Brasil quanto de Portugal, análise de outra categoria.

Sobre a terceira categoria temática, Quantidade de autores por Dossiê, foram identificados no total 50 (100\%) autores, sendo que destes I 3 (26\%), I7 (34\%) e 20 (40\%) autores publicaram seus artigos científicos, respectivamente, nos Dossiês dos anos de 2014, de 2015 e de 2017.

Sobre a quarta categoria temática, Distribuição de autoria única e múltipla, do total de 32 artigos científicos publicados (100\%), I 9 (59\%) artigos científicos foram identificados sob autoria única e I 3 (4I\%) artigos foram identificados sob autoria múltipla. Os resultados quanto à autoria única ou múltipla por Dossiê são apresentados no Quadro I.

Quadro I:Autoria única e múltipla por Dossiê

\begin{tabular}{|c|c|c|c|c|c|c|c|c|}
\hline \multirow{2}{*}{ Autoria } & \multicolumn{2}{|c|}{ Dossiê 20I4 } & \multicolumn{2}{c|}{ Dossiê 20I5 } & \multicolumn{2}{|c|}{ Dossiê 20I7 } & \multicolumn{2}{|c|}{ Total } \\
\cline { 2 - 10 } & $\mathrm{F}$ & $\%$ & $\mathrm{~F}$ & $\%$ & $\mathrm{~F}$ & $\%$ & & \\
\hline Artigos de autoria única & 6 & 32 & 5 & 26 & 8 & 42 & 19 & 100 \\
\hline Artigos de autoria múltipla & 3 & 24 & 5 & 38 & 5 & 38 & 13 & 100 \\
\hline
\end{tabular}

Fonte: elaboração própria - 2018.

Sendo $F=$ Frequência de artigos.

Apesar da autoria múltipla (em sua maior parte formada por autoria dupla) como forma de colaboração científica muitas vezes interinstitucional, nos Dossiês publicados pela RITUR foi destacada a autoria única dos artigos científicos como maioria, principalmente no Dossiê publicado em 2017, com 8 (42\%) de artigos científicos de autoria única.

Sobre a quinta categoria temática, Formação acadêmica dos autores, foram identificadas as seguintes formações acadêmicas ${ }^{7}$ dos 50 (100\%) autores: 37 (74\%) são Doutores (nas áreas de Turismo, Antropologia, Ciência Política, Comunicação, História, História e Filosofia da Ciência, Museologia, História da Arte, Psicologia, Arqueologia, Desenvolvimento Rural, Educação, Literatura, Linguística e Sociologia); 8 (16\%) são Doutorandos (nas áreas de História e Filosofia da Ciência, Ciência da Informação e Turismo); 4 (8\%) são Mestres (nas áreas de Humanidades e Turismo); e I (2\%) é apenas Graduado (na área de Turismo). Sendo assim, evidencia-se que a maioria dos autores possui como maior titulação o Doutorado em áreas diversas, mas destacando-se a área da Museologia, o que é constatado pela relação "Museus, Turismo e Sociedade" proposta pelos Dossiês da RITUR.

Sobre a sexta categoria temática, Instituições em que os autores realizaram a sua formação acadêmica, os resultados são apresentados no Quadro 2.

7 Não se admitiu dupla contagem das áreas de formações acadêmicas dos autores, sendo indicadas as áreas identificadas sem repetições e sem suas especialidades. 
Quadro 2 : Instituições em que os autores realizaram sua formação acadêmica:

\begin{tabular}{l|l|l} 
Instituições & F & $\%$ \\
\hline Universitat de Girona, Espanha & 8 & 16 \\
\hline Universidade de Évora, Portugal & 7 & $\mathrm{I} 4$ \\
\hline Universidade Lusófona de Humanidades e Tecnologias, Portugal & 6 & $\mathrm{I} 2$ \\
\hline Universidade Federal da Bahia, Brasil & 4 & 8 \\
\hline Universidade de São Paulo, Brasil & 4 & 8 \\
\hline Universidad de La Laguna, Espanha & 3 & 6 \\
\hline Universidade de Lisboa, Portugal & 2 & 4 \\
\hline Pontifícia Universidade Católica do Rio Grande do Sul, Brasil & 2 & 4 \\
\hline Université Laval, Canadá & $\mathrm{I}$ & 2 \\
\hline Universidade Federal do Rio Grande do Sul, Brasil & $\mathrm{I}$ & 2 \\
\hline Universidade Federal do Rio de Janeiro, Brasil & $\mathrm{I}$ & 2 \\
\hline Universidade Federal de Santa Catarina, Brasil & $\mathrm{I}$ & 2 \\
\hline Universidade Federal de Minas Gerais, Brasil & $\mathrm{I}$ & 2 \\
\hline Universidade do Vale do Rio dos Sinos, Brasil & $\mathrm{I}$ & 2 \\
\hline Universidade de Coimbra, Portugal & $\mathrm{I}$ & 2 \\
\hline Universidade de Caxias do Sul, Brasil & $\mathrm{I}$ & 2 \\
\hline Universidad de Salamanca, Espanha & $\mathrm{I}$ & 2 \\
\hline Universidad de Las Palmas de Gran Canaria, Espanha & $\mathrm{I}$ & 2 \\
\hline Universidad Autónoma de Madrid, Espanha & $\mathrm{I}$ & 2 \\
\hline Pontifícia Universidade Católica de São Paulo, Brasil & $\mathrm{I}$ & 2 \\
\hline Instituto Universitário de Lisboa, Portugal & $\mathrm{I}$ & 2 \\
\hline Faculdades Integradas da Bahia, Brasil & $\mathrm{I}$ & 2 \\
\hline Total & 50 & 100 \\
\hline Fon & & 2 \\
\hline
\end{tabular}

Fonte: elaboração própria - 2018.

Sendo $F=$ Frequência de instituições em que os autores realizaram sua formação acadêmica.

Evidencia-se a vinculação da formação acadêmica dos autores em renomadas universidades nacionais e estrangeiras, destacando-se a UdG, Espanha, a UÉvora, Portugal e a Universidade Lusófona de Humanidades e Tecnologias (ULHT), Portugal, em sua maioria, pelo fato dessas universidades promoverem seus tradicionais programas de pós-graduação nas áreas de Turismo e Museologia, que coincidem com a temática dos Dossiês da RITUR: o Programa de Doutorado em Turismo da UdG, Espanha; o Programa de Doutoramento em História e Filosofia da Ciência com Especialidade em Museologia da UÉvora, Portugal; e o Programa de Doutoramento em Museologia da ULHT, Portugal. Sobre a sétima categoria temática, Instituições de vinculação dos autores, os resultados são apresentados no Quadro 3. 
Quadro 3: Instituições de vinculação dos autores

\begin{tabular}{l|l|l} 
Instituições & F & $\%$ \\
\hline Estudante de Doutorado, sem vinculação profissional & 4 & 8 \\
\hline Universitat de Girona, Espanha & 4 & 8 \\
\hline Universidade Federal da Bahia, Brasil & 4 & 8 \\
\hline Universidade Lusófona de Humanidades e Tecnologias, Portugal & 3 & 6 \\
\hline Universidade Federal da Paraíba, Brasil & 3 & 6 \\
\hline Universidade de Évora, Portugal & 3 & 6 \\
\hline Universidade de Caxias do Sul, Brasil & 3 & 6 \\
\hline Universidade Federal de Goiás, Brasil & 2 & 4 \\
\hline Universidade Federal de Alagoas, Brasil & 2 & 4 \\
\hline Universidade de São Paulo, Brasil & 2 & 4 \\
\hline Universidade de Brasília, Brasil & 2 & 4 \\
\hline Universidad de La Laguna, Espanha & 2 & 4 \\
\hline Museu d'Art de Girona, Espanha & 2 & 4 \\
\hline Escola Superior de Hotelaria e Turismo de Estoril, Portugal & 2 & 4 \\
\hline Universidade Federal do Pará, Brasil & $\mathrm{I}$ & 2 \\
\hline Universidade Federal do Estado do Rio de Janeiro, Brasil & $\mathrm{I}$ & 2 \\
\hline Universidade Federal de Santa Catarina, Brasil & $\mathrm{I}$ & 2 \\
\hline Universidade Estadual Paulista Júlio de Mesquita Filho, Brasil & $\mathrm{I}$ & 2 \\
\hline Universidade do Algarve, Portugal & $\mathrm{I}$ & 2 \\
\hline Universidade de Coimbra, Portugal & $\mathrm{I}$ & 2 \\
\hline Universidad de Las Palmas de Gran Canaria, Espanha & $\mathrm{I}$ & 2 \\
\hline Universidad Complutense de Madrid, Espanha & $\mathrm{I}$ & 2 \\
\hline Museo Memorial del Exilio, Espanha & $\mathrm{I}$ & 2 \\
\hline Instituto Politécnico de Leiria, Portugal & $\mathrm{I}$ & 2 \\
\hline Faculdade de Tecnologia e Ciências, Brasil & $\mathrm{I}$ & 2 \\
\hline Departamento de Cultura Generalitat de Catalunya, Espanha & $\mathrm{I}$ & 2 \\
\hline Total & 50 & 100 \\
\hline & & 2 \\
\hline
\end{tabular}

Fonte: elaboração própria - 2018.

Sendo F = Frequência de instituições de vinculação dos autores.

Dos 50 (100\%) autores que publicaram artigos científicos nos Dossiês temáticos da RITUR, a maioria, 27 (54\%) autores, possui vinculação institucional estrangeira, de Portugal e Espanha, contra 23 (46\%) autores com vinculação institucional nacional, de acordo com o escopo da RITUR de fomentar a comunicação científica sobre Turismo e áreas afins produzida na América Latina e Europa Ibérica. Destacam-se 4 (8\%) autores estudantes de Doutorado sem vinculação profissional e a UdG, Espanha, como instituição de maior expressão.

Sobre a oitava categoria temática,Temas dos artigos científicos por Dossiê, foram identificados 6 temas a partir dos seguintes elementos dos artigos científicos: título, resumo e palavras-chave e, sobretudo, texto completo. No entanto, esclarece-se que os temas identificados dos artigos científicos não são limitadores de outras possibilidades de leitura, interpretação e classificação. Fo- 
ram os temas identificados: Museu, Memória e Turismo; Museu, Patrimônio e Turismo; Museu e Turismo Cultural; Museu, Tecnologia e Turismo; Museu, Exposição e Turismo; e Produção Científica em Museologia.

Sobre a nona categoria temática, Frequência de Temas dos artigos científicos, seus resultados são apresentados no Quadro 4.

Quadro 4: Frequência de Temas dos artigos científicos

\begin{tabular}{|c|c|c|c|c|c|c|c|c|}
\hline \multirow{2}{*}{ Temas dos Artigos Científicos } & \multicolumn{2}{|c|}{ Dossiê 2014} & \multicolumn{2}{|c|}{ Dossiê 2015} & \multicolumn{2}{|c|}{ Dossiê 2017} & \multicolumn{2}{|c|}{ Total } \\
\hline & $\mathrm{F}$ & $\%$ & $\mathrm{~F}$ & $\%$ & $\mathrm{~F}$ & $\%$ & $\mathrm{~F}$ & $\%$ \\
\hline Museu, Memória e Turismo & 3 & 10 & 4 & 13 & 1 & 3 & 8 & 24 \\
\hline Museu, Patrimônio e Turismo & I & 3 & - & - & 6 & 20 & 7 & 22 \\
\hline Museu e Turismo Cultural & 2 & 6 & 2 & 6 & 2 & 6 & 6 & 20 \\
\hline Museu, Tecnologia e Turismo & 3 & 9 & $\mathrm{I}$ & 3 & I & 3 & 5 & 15 \\
\hline Museu, Exposição e Turismo & - & - & 2 & 6 & 2 & 6 & 4 & 13 \\
\hline Produção Científica em Museologia & - & - & I & 3 & I & 3 & 2 & 6 \\
\hline Total & 9 & 28 & 10 & 31 & 13 & 41 & 32 & 100 \\
\hline
\end{tabular}

Fonte: elaboração própria - 2018.

Sendo $\mathrm{F}=$ Frequência de temas identificados por artigos científicos.

Os temas que mais se destacaram foram: Museu, Memória e Turismo; Museu, Patrimônio e Turismo; e Museu e Turismo Cultural. Sendo que, destes três temas, apenas o tema Museu, Memória e Turismo e o tema Museu, Patrimônio e Turismo se apresentaram em todas as três edições dos Dossiês da RITUR sobre "Museus, Turismo e Sociedade". Outro tema que se apresentou em todas as edições dos Dossiês foi Museu, Tecnologia e Turismo.

Sobre a décima categoria temática, Palavras-chave mais citadas, considerou-se pertinente determinar um valor de corte diante da dispersão e número alto de palavras-chave. Dessa forma, o critério se assentou sobre o fato das palavras-chave terem sido citadas mais de uma vez nos três Dossiês da RITUR. Foram citadas ao todo I 46 (I00\%) palavras-chave nos três Dossiês. Segundo a determinação do valor de corte, as palavras-chave mais citadas foram: Museu(s), com I5 (I0\%) citações; Turismo, com II (8\%) citações; Turismo cultural, com 6 (4\%) citações; Patrimônio Cultural, com 5 (3\%) citações; Museologia, com 4 (2,7\%) citações; Patrimônio, com 4 (2,7\%) citações; Sociedade, com 3 (2\%) citações; e com 2 ( $1 \%$ ) citações cada, têm-se as palavras-chave: Cultura; Ecomuseu; Educação Patrimonial; Estudo de caso; Experiência; e ICOM.

Sobre a décima-primeira categoria temática,Tipologia documental referenciada, foram identificadas II 27 referências utilizadas nos artigos científicos publicados nos três Dossiês da RITUR. Destas, 577 (5I\%) referências se referiram a Livros ou Capítulos de Livro, 333 (30\%) referências se referiram a Artigos de periódicos, 47 (4\%) referências se referiram a Comunicações em eventos, 24 (2\%) referências se referiram a Teses de doutorado e empatados tecnicamente 22 (2\%) referências se referiram a Dissertações de mestrado, além de I 24 (I I\%) referências classificadas como de outro tipo, como documentos on-line, leis, estatutos, relatórios, declarações, projetos, programas de governo, instituições e fontes históricas. Cabe ressaltar a hegemonia da cultura do uso dos Livros ou Capítulos de Livro, seguidos dos artigos de periódicos, pela atualização do conhecimento científico publicado, totalizando as duas tipologias 1010 (8I\%) referências.

E sobre a décima-segunda e última categoria temática, Autores mais referenciados, considerou-se pertinente determinar um valor de corte. Dessa for- 
ma, o critério se assentou sobre o fato de o autor ter sido referenciado pelo menos cinco vezes nos três Dossiês da RITUR.A partir do critério estabelecido, chegou-se a um total de 26 autores. $O$ elenco dos autores, seguida da frequência com que foram referenciados encontra-se no Quadro $5^{8}$ :

Quadro 5:Autores mais referenciados

\begin{tabular}{|l|l|l|l|}
\hline Autores mais referenciados & Área de atuação & País & F \\
\hline ICOM & Museologia & França (sede) & I7 \\
\hline Marília Xavier Cury & Museologia & Brasil & I4 \\
\hline Manuel de Azevedo Antunes & Museologia & Portugal & II \\
\hline Luciana Ferreira da Costa & Ciência da Informação & Brasil & 9 \\
\hline Unesco & Educação, Ciência e Cultura & França (sede) & 9 \\
\hline Teresa Cristina Moletta Scheiner & Museologia & Brasil & 8 \\
\hline Iphan & Patrimônio & Brasil & 7 \\
\hline Richard Prentice & Turismo & Escócia & 7 \\
\hline John Howard Falk & Museologia & EUA & 6 \\
\hline Mário Caneva Moutinho & Museologia & Portugal & 6 \\
\hline MINOM Brasil & Museologia & Brasil (sede) & 6 \\
\hline Alexandra Rodrigues Gonçalves & Turismo & Portugal & 5 \\
\hline Andre Desvalées & Museologia & França & 5 \\
\hline Dominique Poulot & Museologia & França & 5 \\
\hline Hugues de Varine & Museologia & França & 5 \\
\hline Irene Vaquinhas & Museologia & Portugal & 5 \\
\hline Judite Santos Primo & Museologia & Portugal & 5 \\
\hline Llorenç Prats & Turismo & Espanha & 5 \\
\hline Manuelina Maria Duarte Cândido & Museologia & Brasil & 5 \\
\hline Margarita Barretto & Turismo & Brasil & 5 \\
\hline Maria Margaret Lopes & Museologia & Brasil & 5 \\
\hline Mário de Souza Chagas & Museologia & Brasil & 5 \\
\hline Pierre Bourdieu & Sociologia & França & 5 \\
\hline Susana de Araújo Gastal & Turismo & Brasil & 5 \\
\hline OMT & Turismo & Espanha & 5 \\
\hline Zygmunt Bauman & Sociologia & Polônia & 5 \\
\hline Fonte & & \\
\hline
\end{tabular}

Fonte: elaboração própria - 2018.

Sendo $F=$ Frequência de referências encontradas.

Dentre os autores mais referenciados, destacam-se: o ICOM, como autor institucional dedicada à área da Museologia; Marília Xavier Cury, Professora do Programa de Pós-Graduação Interunidades em Museologia (PPGMus) da Universidade de São Paulo (USP), Brasil; Manuel de Azevedo Antunes, Professor do Programa de Doutoramento em Museologia da ULHT, Portugal; Luciana Ferreira da Costa, Líder responsável pela REDMUS do PPGCI da UFPB, Brasil; e a UNESCO, também como autora institucional, dedicada às áreas da Educação, Ciência e Cultura.

8 Como não foram analisados os autores mais referenciados em relação ao total encontrado, não foram utilizados percentuais no Quadro 5. 


\section{Considerações Finais}

Entende-se que neste artigo foi alcançado o objetivo da realização de uma análise bibliométrica da produção científica sobre "Museus, Turismo e Sociedade" dos artigos científicos de três Dossiês dedicados a esta temática e publicados pela RITUR nos anos de 20I4,20I5 e 2017.

Como estudo interdisciplinar entre os estudos de museus e Turismo, com o aporte da bibliometria de origens na Biblioteconomia e Ciência da Informação e a análise de conteúdo por categorias, entende-se, também, que este artigo conferiu a possibilidade de atribuir sentido aos resultados encontrados, qualificando-os para que possam ter melhor uso por parte de políticas de C\&T, centros/grupos de pesquisas, nos diálogos interdisciplinares aos transdisciplinares entre as áreas da Museologia e do Turismo, como se propôs a RITUR em seus Dossiês. Neste sentido, os Dossiês se demonstraram contributivos para sanar uma lacuna sobre o pequeno número de publicações sobre a temática proposta e como canal de comunicação científica disposto a veiculá-las, numa dimensão internacional, na perspectiva do que é produzido na América Latina e Europa lbérica na contemporaneidade.

Sendo assim, este artigo acompanha a vertente de trabalhos atuais realizados por autores da Ciência da Informação que realizam uma leitura das pesquisas bibliométricas à luz de elementos do contexto sócio-histórico em que a atividade científica é produzida.

Conclui com a importância das relações entre museus e Turismo na sociedade contemporânea e a contribuição dos Dossiês da RITUR para a compreensão e o avanço dessas relações, em perspectivas teórico-epistemológicas, práticas e políticas. Os temas levantados nos artigos científicos dos Dossiês demonstram muito bem tal situação, apresentando-se como tendências temáticas essenciais para a compreensão das relações entre museus e Turismo também com outras áreas de conhecimento, sendo, ademais, indicativos das frentes de investigação da produção científica no diálogo dessas áreas no século XXI.

Por fim, cabe ressaltar que está previsto para o final do segundo semestre do ano de 2018 a publicação do Dossiê Número 4 sobre "Museus, Turismo e Sociedade" da RITUR, como compromisso contínuo deste periódico científico com a demanda crescente da comunidade científica sobre esta temática, sob os preceitos do ICOM, da FMAM e da OMT (informação verbal).

\section{Referências}

AGENCIA ESPAÑOLA DE COOPERACIÓN INTERNACIONAL PARA EL DESARROLLO (AECID).AECID. Madrid, 20 I8. Disponível em: http://www.aecid.es/ EN/aecid. Acesso em: 06 jun. 2018.

ALBARELLO, Luc et al. Práticas e métodos de investigação em Ciências Sociais. Lisboa: Gradiva, 1997.

ARAÚJO, Carlos Alberto Ávila. Bibliometria: evolução histórica e questões atuais. Em Questão, v. I2, n. I, p. I I-32, 2006.

BARDIN, Laurence. Análise de conteúdo. Lisboa, Edições 70, 1979.

BRINKMAN, Manus. Museums Heritage and Cultural Tourism - Closing Speech. 
Secretary General of ICOM. Paris: ICOM, 2000, p. 85-86.

COORDENAÇÃO DE APERFEIÇOAMENTO DE PESSOAL DE NÍVEL SUPERIOR (CAPES). Qualis Periódicos. Plataforma Sucupira. Brasília, 20I8. Disponível em: https://sucupira.capes.gov.br/sucupira/public/consultas/coleta/veiculoPublicacaoQualis/listaConsultaGeralPeriodicos.jsf. Acesso em: 06 jun. 2018.

COSTA, Luciana Ferreira da; NUNES, Maria de Fátima. Terceiro encontro de olhares sobre Museus, Turismo e Sociedade. Revista Iberoamericana de Turismo, Penedo, v. 7, Dossiê n. 3, 2017, p. I-5. Disponível em: http://www.seer.ufal.br/ index.php/ritur/article/view/4I70/30I2.Acesso em: 06 jun. 2018.

COSTA, Luciana Ferreira da; NUNES, Maria de Fátima; LOPES, Maria Margaret. Scientific electronics journals from Brazil in Museology. In: INTERNATIONAL CONFERENCE ON INFORMATION SYSTEMS AND TECHNOLOGY MANAGEMENT - CONTECSI, I2., 20I5, São Paulo. Proceedings... São Paulo: USP, 20I5, p. 4663-4677.

CRESPO, Isabel Merlo. Um estudo sobre o comportamento de busca e uso de informação de pesquisadores das áreas de Biologia Molecular e Biotecnologia: impactos do periódico científico eletrônico. Dissertação (Mestrado em Comunicação). Universidade Federal do Rio Grande do Sul, Porto Alegre, 2005.

GONÇALVES, Alexandra Rodrigues. Museus, Turismo e Sociedade - uma reflexão. Revista lberoamericana de Turismo, Penedo, v. 7, Dossiê n. 3, p. 26-67, 2017.

INSTITUTO BRASILEIRO DE INFORMAÇÃO EM CIÊNCIA ETECNOLOGIA (IBICT). Sistema Eletrônico de Editoração de Revistas (SEER). Brasília, 20I8. Disponível em: http://www.ibict.br/pesquisa-desenvolvimento-tecnologico-e-inovacao/sistema-eletronico-de-editoracao-de-revistas-seer.Acesso em: 06 jun. 2018.

INSTITUTO DE HISTÓRIA CONTEMPORÂNEA - GRUPO DE INVESTIGAÇÃO CIÊNCIA, ESTUDOS DE HISTÓRIA, FILOSOFIA E CULTURA CIENTÍFICA (IHC-CEHFCI). História. Évora, 2018. Disponível em: http://www.iifa.uevora. $\mathrm{pt/galeria/galeria \_ videos/Centros-de-Investigacao-e-Catedras/CEHFCi-Centro-}$ -de-Estudos-de-Historia-e-Filosofia-da-Ciencia. Acesso em:06 jun. 2018.

INTERNATIONAL COUNCIL OF MUSEUMS (ICOM). Declaration of the International Council of Museums (ICOM) and the World Federation of Friends of Museums (WFFM) for worldwide Sustainable Cultural Tourism (December 2007). Paris, 2007. Disponível em: http://archives.icom.museum/declaration_ tourism_eng.html.Acesso em: 06 jun. 2018.

MAIA, Luís Cláudio Gomes. Estudo de uso de periódicos: Portal Periódicos Capes na UFMG. Dissertação (Mestrado em Ciência da Informação). Universidade Federal de Minas Gerais, Belo Horizonte, 2005.

MARCONI, Marina de Andrade; LAKATOS, Eva Maria. Metodologia do Trabalho Cientifico. São Paulo:Atlas, 2009.

MUELLER, Suzana Pinheiro Machado. A comunicação científica e o movimento 
Museus, Turismo, Sociedade:

análise bibliométrica da produção científica íbero-americana

de acesso livre ao conhecimento. Ciência da Informação, v. 35, n. 2, p. 27-38, 2006.

OBSERVATÓRIOTRANSDISCIPLINAR DE PESQUISAS EMTURISMO (OTPT). Repercussões. 2018. Disponível em: http://www.dgp.cnpq.br/dgp/espelhogrupo/877080 I7094I078I.Acesso em: 06 jun. 2018.

OPEN ARCHIVES INITIATIVE (OAI). Standards for Web Content Interoperability. 2018. Disponível em: https://www.openarchives.org/. Acesso em: 06 jun. 2018.

REDE DE PESQUISA E (IN)FORMAÇÃO EM MUSEOLOGIA E PATRIMÔNIO (REDMUS). Repercussões. 2018. Disponível em: http://dgp.cnpq.br/dgp/espelhogrupo/628527572I 3 I 0405. Acesso em: 06 jun. 2018.

REVISTA IBEROAMERICANA DE TURISMO (RITUR). Diretrizes para Autores. 2018a. Disponível em: http://www.seer.ufal.br/index.php/ritur/about/ submissions\#authorGuidelines. Acesso em: 06 jun. 2018.

REVISTA IBEROAMERICANA DE TURISMO (RITUR). Edições Anteriores. 20l8b. Disponível em: http://www.seer.ufal.br/index.php/ritur/issue/archive. Acesso em: 06 jun. 2018.

REVISTA IBEROAMERICANA DE TURISMO (RITUR). Equipe Editorial. 20I8c. Disponivel em: http://www.seer.ufal.br/index.php/ritur/issue/archive.Acesso em: 06 jun. 2018.

REVISTAIBEROAMERICANA DETURISMO (RITUR).Indexação.20 I8d.Disponível em:http://www.seer.ufal.br/index.php/ritur/about/editorialPolicies\#custom-0. Acesso em: 06 jun. 2018.

REVISTA IBEROAMERICANA DETURISMO (RITUR). Site oficial. 20I8e. Disponível em: http://www.seer.ufal.br/index.php/ritur.Acesso em: 06 jun. 2018.

SANTOS, Raimundo Nonato Macedo dos; KOBASHI, Nair Yumiko. Bibliometria, Cientometria, Infometria: conceitos e aplicações. Pesquisa Brasileira em Ciência da Informação e Biblioteconomia, v. 2, n. I, p. I55-I72, 2009.

SCHEINER, Teresa Cristina Moletta. Reflexões sobre Museus, Turismo, Patrimônio e Sociedade. Revista Iberoamericana de Turismo, Penedo, v. 7, Dossiê n. 3, p. $6-25,2017$.

UNIVERSITAT DE GIRONA (UDG). Facultat de Turisme. Girona, 20 I8. Disponível em: https://www.udg.edu/ca/ft 